\title{
Non-Linear Control Strategies for Attitude Maneuvers in a CubeSat with Three Reaction Wheels
}

\author{
Brayan Espinoza García ${ }^{1}$, Ayrton Martin Yanyachi ${ }^{2}$, Pablo Raúl Yanyachi ${ }^{3}$ \\ Electronic Engineering Professional School, Universidad Nacional de San Agustin, Arequipa, Peru ${ }^{1}$ \\ Pedro Paulet Astronomical and Aerospace Institute, Universidad Nacional de San Agustin, Arequipa, Peru ${ }^{3}$ \\ Vrije Universiteit Brussel, Brussels, Belgium ${ }^{2}$
}

\begin{abstract}
Development of nanosatellites with CubeSat standard allow students and professionals to get involved into the aerospace technology. In nanosatellites, attitude plays an important role since they can be affected by various disturbances such as gravity gradient and solar radiation. These disturbances generate a torque in the system that must be corrected in order to maintain the CubeSat behavior. In this article, the kinematic and dynamic equations applied to a CubeSat with three reaction wheels are presented. In order to provide a solution to the attitude maneuvering problem, three robust control laws developed by Boskovic, Dando, and Chen are presented and evaluated. Furthermore, these laws are compared with a feedback control law developed by Schaub and modified to use Quaternions. The simulated system was subjected to disturbances caused by a Gravity Gradient Torque and misalignments in the reaction wheels. The effectiveness of each law is determined using the Average of Square of the Commanded Control Torque (ASCCT), the Error Euler Angle Integration (EULERINT), the settlement time, the estimated computational cost $(O)$, and the steady-state error $\left(e_{s s}\right)$.
\end{abstract}

Keywords-Attitude control; attitude maneuvers; adaptive control; feedback control; CubeSat; Quaternions; reaction wheels; comparison

\section{INTRODUCTION}

The CubeSat standard, developed in 1999, is intended to reduce development time and costs, as well as increase accessibility to space for students and teachers [1]. As the control of attitude maneuvers is a key factor in the performance of a CubeSat, the aim of this research is to compare and choose the most adequate control law to perform rest-to-rest maneuvers under constant and non-linear disturbances.

Sidi [2], Slotine [3], and Márquez [4] defined the attitude problem as a system of two non-linear matrix equations. Among the attitude system controllers, the one presented by Sidi in [2] is observed. Sidi linearized the attitude equations for small angles and applied PID controllers using Euler Angles and Quaternions. However, this controller was not effective under external disturbances. Control algorithms have also been developed for non-linear systems. These include a feedback control developed by Schaub in [5] where Modified Rodrigues Parameters (MRP) are used to describe the attitude of a satellite, and a Variable Speed Control Moment Gyro (VSCMG) is used as an actuator. Although Schaub's control algorithm had a quick response to constant errors of the inertia tensor, it did not guarantee a quick response to external disturbances. Adaptive control laws have also been developed following the guidelines described by Slotine in [3]. Also, Dando [6] proposed an adaptive control law based on sliding surfaces that calculated the error between the real and the calculated inertia tensor instead of estimating the tensor of inertia itself as proposed by Ahmed in [7]. Nevertheless, this method was only valid for a constant error. Scarritt in [8] estimated a gain applied to the modeled inertia tensor and a rotation associated with the misalignment of the reaction wheel obtaining a robust control algorithm with a high computational cost $(\mathrm{O})$.

All the controllers mentioned above neglected the input control constraints. However, Boskovic [9] focused on evaluating stability under saturation inputs obtaining a robust control algorithm based on the variable structure control. Boskovic's controller was independent of the inertia tensor and has a lower computational cost $(\mathrm{O})$ than the average robust algorithms but its disturbance rejection property cannot be observed. Similarly, Chen [10] developed a robust controller that considered input constraints based on the fast non-singular terminal sliding mode surface (FNTSMS). This controller needed an inertia a priori information but was capable to reject nonlinear disturbances and to keep the tracking error around zero. However, only non-asymptotic stability was guaranteed in a finite time.

This article is divided into the following sections: Section II presents the dynamic and kinematic equations for a rigid body. In Section III, the equations that describe the attitude for a CubeSat with three reaction wheels are introduced. Sections IV and $\mathrm{V}$ present the equations necessary to simulate the gravity gradient torque and the internal disturbances torques produced by reaction wheels misalignment. Section VI presents a bibliographic review of adaptive control laws developed by Dando, Boskovik, and Chen. Also, a modification of the control law developed by Schaub to use three reaction wheels instead of VSCMG and Quaternions instead of MRP is presented. In Section VII, simulations subject to the aforementioned perturbations and to a miscellaneous torque were performed. In order to measure the effectiveness of the control algorithm, a comparison was made applying five criteria. The first criterion of comparison is the steady-state error $\left(e_{s s}\right)$, the second is the Error Euler Angle Integration (EULERINT) used by Sidi in [2], the third is the Average of Square of the Commanded Control Torque (ASCCT) presented by Xiao in [12], the fourth 
is the settlement time, and the fifth is the estimated computational cost $(\mathrm{O})$. Finally, the results, conclusions, and future work are shown in Sections VIII, IX, and X, respectively.

\section{Attitude Equations}

Two equations are required to describe the attitude of a rigid body. First, the kinematic equation that describes the motion of the satellite itself without taking into account the action of forces. Second, the dynamic equation that takes into account the torques acting on the body.

\section{A. Kinematics Equation}

The kinematics can be described using Euler Angles, Rodrigues Parameters, Modified Rodrigues Parameters, Quaternions, and other parameters derived from these. In this article, Quaternions are used for the description of attitude [13] [14].

The kinematic equation for rotations between $0^{\circ}$ to $360^{\circ}$ is defined in (1):

$$
\dot{q}=\frac{1}{2} \Xi(q) \omega
$$

where ' $\Xi(q)$ ' is defined in (2).

$$
\Xi(q) \equiv\left[\begin{array}{ccc}
q_{4} & -q_{3} & q_{2} \\
q_{3} & q_{4} & -q_{1} \\
-q_{2} & q_{1} & q_{4} \\
-q_{1} & -q_{2} & -q_{3}
\end{array}\right]
$$

' $q$ ' is a Quaternion that describes the rotation of the inertial frame to the body frame and has the following form:

$$
q=\left[\begin{array}{llll}
q_{1} & q_{2} & q_{3} & q_{4}
\end{array}\right]^{T}
$$

A Quaternion ' $q$ ' has a scalar part denoted by ' $q_{4}$ ' and a vector part denoted by ' $q_{13}=\left[q_{1}, q_{2}, q_{3}\right]^{T}$ '. Also, ' $q$ ' must have a unit norm to describe a pure rotation, as seen in (4).

$$
\|q\|=q_{1}^{2}+q_{2}^{2}+q_{3}^{2}+q_{4}^{2}=1
$$

\section{B. Dynamics Equation}

The dynamics of the CubeSat, neglecting the effect of the reaction wheels and modeled as a rigid body, is described by (5) [17].

$$
\dot{\omega}=\tilde{J}_{B}^{-1}\left[L-\omega \times \tilde{J}_{B} \omega\right]
$$

Where:

- $\omega$ : Angular velocity of the satellite relative to the inertial frame.

- $\tilde{J}_{B}$ : Body inertia tensor.

- $\quad L$ : External torque applied to the center of mass expressed in the body frame.

All parameters in (5) are expressed in the body frame according to [2], [4] and [16].

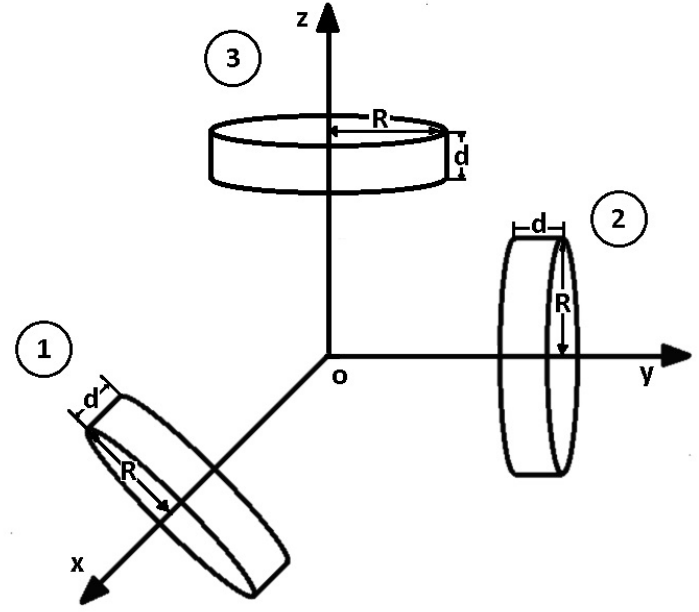

Fig. 1. Distribution of the reaction wheels described in the body frame which origin coincides with the center of mass and geometric. The loads are considered to be fully balanced around the 'o' origin. Adapted from [2]

\section{Three Reaction wheels CubeSat Model}

To consider the effects that reaction wheels have on the system, a CubeSat is simulated with three reaction wheels with the distribution shown in Fig. 1.

' $\tilde{J}_{B}$ ' is defined as the inertia tensor without contributions from the reaction wheels and the general inertia tensor ' $J_{B}$ ' is defined in (6) according to [4].

$$
J_{B} \equiv \tilde{J}_{B}+\sum_{l=1}^{n} J_{l}^{\perp}\left(I_{3}-W_{l} W_{l}\right)^{T} \quad l=1,2,3
$$

Where:

- $W_{l}$ : Rotation axes.

- $J_{l}^{\perp}$ : Reaction wheel inertia perpendicular to the axis of rotation.

- $J_{l}^{\|}$: Reaction wheel inertia parallel to the axis of rotation.

- $I_{3}: 3 \times 3$ Diagonal identity matrix.

Decomposing (6) and taking into account the distribution of the reaction wheels in Fig. 1, the general inertia tensor is defined by (7).

$$
J_{B}=\left[\begin{array}{ccc}
\tilde{J}_{x x}+2 J^{\perp} & -\tilde{J}_{x y} & -\tilde{J}_{x z} \\
-\tilde{J}_{y x} & \tilde{J}_{y y}+2 J^{\perp} & -\tilde{J}_{y z} \\
-\tilde{J}_{z x} & -\tilde{J}_{z y} & \tilde{J}_{z z}+2 J^{\perp}
\end{array}\right]
$$

In the case of reaction wheels with radius ' $R_{r w}$ ', thickness ' $d_{r w}$ ' and mass ' $m_{r w}$ ', the parallel and perpendicular inertias are defined by (8) and (9), respectively.

$$
J^{\|}=\frac{1}{2} m_{r w} R_{r w}^{2}
$$

$$
J^{\perp}=\frac{1}{4} m_{r w} R_{r w}^{2}+\frac{1}{12} m_{r w} d_{r w}^{2}
$$


The dynamics of the satellite is defined in (10) according to [2] and [4].

$$
\dot{\omega}=J_{B}^{-1}\left[L-L_{r w}-\omega \times\left(J_{B} \omega+H_{B}^{w}\right)\right]
$$

Where:

- $\quad L$ : External torque applied to the center of mass expressed in the body frame.

- $\quad L_{r w}$ : Torque delivered by reaction wheels.

- $H_{B}^{w}$ : Angular momentum delivered by the reaction wheels.

Equation (6) excludes the inertia of the reaction wheels parallel to the rotation axis since this is considered as a body that rotates freely when the reaction wheel is turned off. However, the parallel inertias are taken into account when the general angular momentum of the system is evaluating (11).

The angular momentum delivered by the reaction wheels is defined by (11):

$$
H_{B}^{w}=\sum_{l=1}^{n} J_{l}^{\|}\left(W_{l} \omega+\Omega_{l}\right) W_{l} \quad l=1,2,3
$$

where $\Omega_{l}$ represents the angular speed of the reaction wheels.

Particularly, (11) is expressed as follows:

$$
H_{B}^{w}=J_{r w}[\omega+\Omega]
$$

where $J_{r w}$ is defined by (13).

$$
J_{r w}=\left[\begin{array}{ccc}
J^{\|} & 0 & 0 \\
0 & J^{\|} & 0 \\
0 & 0 & J^{\|}
\end{array}\right]
$$

The torque produced by the reaction wheels can be calculated with (14).

$$
L_{r w}=J_{r w}(\dot{\omega}+\dot{\Omega})
$$

With (12) and (13), the dynamics described in (10) can be rewritten as (15).

$$
\dot{\omega}=J_{B}^{-1}\left[L-L_{r w}-\omega \times\left(J_{B} \omega+J_{r w}[\omega+\Omega]\right)\right]
$$

Finally, the attitude for a CubeSat with three reaction wheels is fully modeled with (1) and (15), the acceleration of the reaction wheels can also be calculated with (16).

$$
\dot{\Omega}=J_{r w}^{-1} L_{r w}-\dot{\omega}
$$

\section{EXTERNAL DISTURBANCES}

\section{A. Gravity Gradient Torque}

The external torques that affect a CubeSat are various. However, for low orbit satellites, the major disturbance that a CubeSat is exposed to is the gravity gradient torque ' $L_{g g}$ ' which is defined in [13] and [19] as (17).

$$
L=L_{g g}=3 \omega_{o}^{2} c_{3} \times J_{B} c_{3}
$$

And ' $\omega_{o}$ ' and ' $c_{3}$ ' is defined in (18) and (19), respectively.

$$
\omega_{o}^{2}=\frac{\mu}{r_{c}^{3}}
$$

$$
c_{3}=C(q)\left[\begin{array}{l}
0 \\
0 \\
1
\end{array}\right]=\left[\begin{array}{l}
2\left(q_{1} q_{3}-q_{2} q_{4}\right) \\
2\left(q_{2} q_{3}+q_{1} q_{4}\right) \\
1-2\left(q_{1}^{2}-q_{2}^{2}\right)
\end{array}\right]
$$

Where:

- $\mu$ : Earth's gravitational coefficient $\left(\mu=3.986 \times 10^{14}\right)$.

- $r_{c}^{3}$ : Distance from the center of earth.

- $\quad C(q)$ :Attitude Matrix.

- $c_{3}$ : Nadir-pointing unit vector.

\section{REACTION WHEELS DISTURBANCES}

Among the most common disturbances, the friction presented in the motors of the reaction wheels [11] and the misalignments [12] are considered.

\section{A. Reaction Wheel Misalignments}

The reaction wheel configuration presented in Fig. 1 is an ideal configuration. In practice, the torque produced by the misalignments is modeled as shown in Fig. 2.

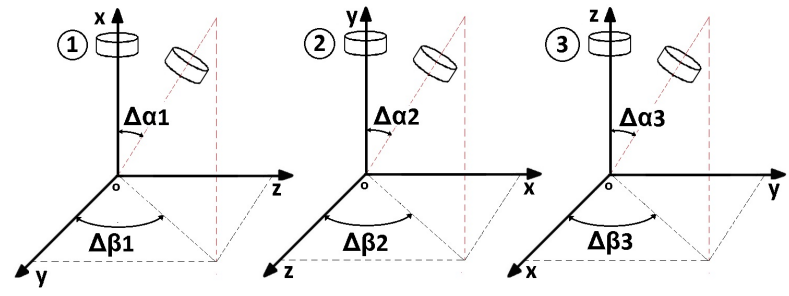

Fig. 2. Misalignments in the reaction wheels. ' $\Delta \alpha_{1}$ ', ' $\Delta \alpha_{2}$ ', ' $\Delta \alpha_{3}$ ' are small angles and ' $\Delta \beta_{1}$ ', ' $\Delta \beta_{2}$ ', ' $\Delta \beta_{3}$ ' are in the range of ' $[-p i, p i]$ '. Adapted from [12].

The equation (20) describes the total torque produced by the reaction wheels when the misalignment angles are small.

$$
L_{r w}^{*}=L_{r w}+\Delta D L_{r w}
$$

Where:

$$
\Delta D=\left[\begin{array}{ccc}
0 & \Delta \alpha_{2} \sin \left(\Delta \beta_{2}\right) & \Delta \alpha_{3} \cos \left(\Delta \beta_{3}\right) \\
\Delta \alpha_{1} \cos \left(\Delta \beta_{1}\right) & 0 & \Delta \alpha_{3} \sin \left(\Delta \beta_{3}\right) \\
\Delta \alpha_{1} \sin \left(\Delta \beta_{1}\right) & \Delta \alpha_{2} \cos \left(\Delta \beta_{2}\right) & 0
\end{array}\right]
$$

The angles ' $\Delta \alpha_{1}$ ', ' $\Delta \alpha_{2}$ ', ' $\Delta \alpha_{3}$ ', ' $\Delta \beta_{1}$ ', ' $\Delta \beta_{2}$ ', ' $\Delta \beta_{3}$ ' are defined in Fig. 2.

\section{Control Laws}

In this article, three control laws for attitude maneuvers are compared. In order to maintain consistency throughout the article, the nomenclatures of each author were changed to those defined in [13] by Markley \& Crassidis as shown in Appendix A (Table VI). The ' $u$ ' control signal becomes the torque delivered by reaction wheels ' $L_{r w}$ ' from (10).

Before starting to describe the control laws, first define the Quaternion error ' $\delta q$ ' between the CubeSat's attitude ' $q$ ' and the desired attitude ' $q_{d}$ ' according to Scarritt [8].

$$
\delta q=\left[\begin{array}{c}
\delta q_{13} \\
\delta q_{4}
\end{array}\right]
$$


Where:

$$
\begin{array}{r}
\delta q_{13}=\Xi^{T}\left(q_{d}\right) q \\
\delta q_{4}=\left(q_{d}\right)^{T} q
\end{array}
$$

And the angular velocity error is defined as ' $\delta \omega=\omega-\omega_{d}$ '. Where ' $\omega$ ' is the angular velocity of the CubeSat relative to the inertial frame expressed in the body frame and ' $\omega_{d}$ ' is the desired angular velocity expressed in the frame of the body.

In most cases, only the desired angular velocity expressed in the inertial frame ' $\omega_{d R}$ ' is available. Then, the rotation matrix ' $C(\delta q)$ ' is needed to express it in the body frame [6].

$$
\delta \omega=\omega-C(\delta q) \omega_{d R}
$$

The skew-symmetric matrix is also defined in (25) [17].

$$
[\omega \times]=\left[\begin{array}{ccc}
0 & -\omega_{3} & \omega_{2} \\
\omega_{3} & 0 & -\omega_{1} \\
-\omega_{2} & \omega_{1} & 0
\end{array}\right]
$$

\section{A. Quaternion Feedback Controller}

The tracking problem is defined by the error variables ' $\delta \omega$ ' and ' $\delta \beta$ ' defined in (26).

$$
\delta \beta=q_{13}-q_{d 13}
$$

The control law is defined in (27).

$$
\begin{aligned}
u=P \delta \omega+K \delta \beta-[\omega \times]\left(J_{B} \omega-\right. & \left.J_{r w}(\omega+\Omega)\right) \\
& -J_{B}\left(\dot{\omega}_{d}-[\omega \times] \omega_{d}\right)
\end{aligned}
$$

Where ' $\mathrm{K}$ ' and ' $\mathrm{P}$ ' are positive definite matrices. Lyapunov's candidate function is defined by Schaub in [5] as (28).

$$
V=\frac{1}{2} \delta \omega^{T} J_{B} \delta \omega+K \delta \beta^{T} \delta \beta
$$

The dynamics of the closed-loop model is defined by (29).

$$
J_{B} \frac{d^{B}}{d t} \delta \omega+K \beta+P \delta \omega=0
$$

Where $\frac{d^{B}}{d t}$ is the derivative with respect to the body frame. By deriving (28) and replacing it with (29), (30) is described as follows:

$$
\begin{aligned}
\dot{V}=\delta \omega^{T}\left[u-[\omega \times] J_{B} \omega\right. & -[\omega \times] J_{r w}(\omega+\Omega) \\
& \left.-J_{B} \dot{\omega}_{d}+J[\omega \times] \omega_{d}+K \delta \beta\right]
\end{aligned}
$$

1) Stability Proof: By replacing (27) in (30), (31) is obtained.

$$
\dot{V}=-\delta \omega^{T}[P] \delta \omega
$$

It can be seen that (31) is a positive semi-defined function. Thus, the system is stable. However, since (28) is dependent on two variables, asymptotically stability can not be ensured.

Nevertheless, using the Mukherjee and Chen theorem [18] we can show that the closed-loop system is asymptotically stable. However, it is important to mention that the value of the calculated inertia tensor will never be equal to the real inertia tensor. Hence, the equation (27) is rewritten as (32).

$$
\begin{array}{r}
u=P \delta \omega+K \delta \beta-[\omega \times]\left(J_{B}^{*} \omega-J_{r w}^{*}(\omega+\Omega)\right) \\
-J_{B}^{*}\left(\dot{\omega}_{d}-[\omega \times] \omega_{d}\right)
\end{array}
$$

Where ' $J_{B}^{*}$ ' and ' $J_{r w}^{*}$ ' are the calculated inertia tensor and the matrix of parallel inertias calculated for the reaction wheels, respectively.

\section{B. Boskovic Robust Controller}

Boskovic's work [9] is based in the variable structure approach and his control technique does not require previous knowledge of the inertia tensor. In addition, Boskovic designed an adaptive gain that allows to compensate the disturbances torques and to ensure that attitude and angular velocity errors will tend to zero.

The control law proposed by Boskovic is given by (33).

$$
-u_{i}(t)=-u_{\max } \frac{s_{i}(t)}{\left|s_{i}(t)\right|+k^{2}(t) \delta_{k}} \quad i=1,2,3
$$

Where ' $\delta_{k}$ ' is a positive constant, ' $\mathrm{K}(\mathrm{t})$ ' is the adaptive gain and ' $u_{\max }$ ' is the torque limit for all control torques. The Boskovic Sliding Vector ' $s(t)$ ' is defined by (34).

$$
s(t)=\delta \omega(t)+k^{2} \delta q_{13}(t)
$$

Boskovic defined, in [9], the adjustment law for the timevarying control gain as (35).

$$
\begin{gathered}
\dot{k}(t)=\frac{\gamma k(t)}{1+4 \gamma\left[1-\delta q_{4}(t)\right]}\left\{u _ { \operatorname { m a x } } \sum _ { i = 1 } ^ { 3 } \left[\frac{\delta \omega_{i} \delta q_{i}(t)}{\left|s_{i}(t)\right|+k^{2}(t) \delta_{k}}-\right.\right. \\
\left.\left.\frac{\left|\delta \omega_{i}(t)\right|\left(1+\delta_{k}\right)}{\left|\delta \omega_{i}(t)\right|+k^{2}(t)\left(1+\delta_{k}\right)}\right]-\delta \omega^{T} \delta q_{13}-k^{2} \delta q_{13}^{T} \delta q_{13}\right\}
\end{gathered}
$$

where ' $\gamma$ ' is a positive scalar and is called the convergence rate.

\section{Dando Adaptive Controller}

The adaptive control law proposed by Dando [6] is intended to estimate the error of the inertia tensor instead of the inertia tensor itself.

The closed-loop error dynamics are first defined in (36).

$$
J_{B} s \dot{(t)}=-[\omega(t) \times] J_{B} \omega(t)-J_{B} \alpha_{r}(t)+u(t)
$$

The Dando Sliding Vector is defined in (37).

$$
s(t)=\omega(t)-\omega_{r}(t)
$$

And the other parameters are defined as:

$$
\begin{gathered}
\alpha_{r}(t)=C(\delta q) \dot{\omega}_{d R}-[\delta \omega \times] C(\delta q) \dot{\omega}_{d R}-\lambda \delta \dot{q}_{13} \\
\omega_{r}=C(\delta q) \omega_{d R}-\lambda \operatorname{sgn}\left[\delta q_{4}\right] \delta q_{13}
\end{gathered}
$$

In this control, a priori knowledge of the inertia tensor with a certain level of uncertainty is assumed as defined in (40).

$$
J_{B}=J_{B}^{*}+\tilde{J}_{E}
$$

Where ' $J_{B}$ ' is the real satellite inertia tensor and ' $J_{B}^{*}$ ' is the calculated inertia tensor and ' $\tilde{J}_{E}$ ' is the inertia uncertainty. 
The control law is given by (41) and (42).

$$
\begin{gathered}
-u=-K_{D} s(t)+J_{B}^{*} \alpha_{r}(t)+[\omega(t) \times] J_{B}^{*} \omega(t)+\tilde{u}(t) \\
\tilde{u}(t)=-\tilde{J}_{E} \alpha_{r}(t)-\left[\omega_{r}(t) \times\right] \tilde{J}_{E} \omega(t)
\end{gathered}
$$

Equation (42) is a torque related to the uncertainty inertia tensor. In order to develop an adaptive control, Dando parametrized ' $\tilde{u}(t)$ ' in terms of ' $\tilde{\theta}$ ' defined by (43).

$$
\tilde{\theta}(t)=\left[\tilde{J}_{E_{x x}}, \tilde{J}_{E_{y y}}, \tilde{J}_{E_{z z}}, \tilde{J}_{E_{y z}}, \tilde{J}_{E_{x z}}, \tilde{J}_{E_{x y}}\right]^{T}
$$

Dando in [9], and Ahmed in [7] introduced the ' $L_{o p}$ ' operator defined by (44).

$$
L_{o p}(a)=\left[\begin{array}{cccccc}
a_{1} & 0 & 0 & 0 & a_{3} & a_{2} \\
0 & a_{2} & 0 & a_{3} & 0 & a_{1} \\
0 & 0 & a_{3} & a_{2} & a_{1} & 0
\end{array}\right]
$$

Applying (44) and (43) to parameterize (42) the following equation is obtained:

$$
\tilde{u}(t)=\Phi^{T} \tilde{\theta}(t)
$$

Where ' $\Phi$ ' is defined as (46).

$$
\Phi\left(\omega, \omega_{r}, \alpha_{r}\right)=-\left(L_{o p}\left(\alpha_{r}\right)+\left[\omega_{r} \times\right] L_{o p}(\omega)\right)^{T}
$$

And the adaptive law to estimate ' $\dot{\tilde{\theta}}$ ' is given by (47).

$$
\dot{\tilde{\theta}}(t)=-\Gamma \Phi s(t)
$$

\section{Chen Robust Controller}

The robust control law proposed by Chen [10] is based on the Fast Non-singular Terminal Sliding Mode Surface (FNTSMS) method and adaptive control methods to compensate the inertia tensor uncertainties under constraints in the reaction wheels.

The Chen Sliding Vector is defined in (48).

$$
S=\left[S_{1}, S_{2}, S_{3}\right]^{T}=\delta \omega+\alpha_{1} \delta q_{13}+\alpha_{2} \beta\left(\delta q_{13}\right)
$$

In (48), ' $\alpha_{1}$ ', ' $\alpha_{2}$ ' are positive constants and the ' $\beta$ ' operator is defined in (49) and (50).

$$
\begin{aligned}
& \beta\left(\delta q_{i}\right)= \begin{cases}\operatorname{sig}^{\gamma}\left(\delta q_{i}\right) & \left|\delta q_{i}\right|>\eta \\
r_{1}\left(\delta q_{i}\right)+r_{2} \operatorname{sgn}\left(\delta q_{i}\right) \delta q_{i}^{2} & \left|\delta q_{i}\right| \leq \eta\end{cases} \\
& \operatorname{sig}^{\gamma}\left(\delta q_{i}\right)=\operatorname{sgn}\left(\delta q_{i}\right)\left|\delta q_{i}\right|^{\gamma} \quad i=1,2,3
\end{aligned}
$$

Where ' $r_{1}=(2-\gamma) \eta^{(\gamma-1)}$, ' $r_{2}=(\gamma-1) \eta^{(\gamma-2)}$ ', ' $\gamma>0$ ', ' $\eta<1$ ' and ' $\operatorname{sgn}($.$) ' is the sign function.$

The system to compensate the actuator saturation is defined in (51).

$$
\dot{\zeta}= \begin{cases}0 & \|\zeta\|_{2} \leq \zeta_{0} \\ -k_{1} \zeta-k_{2} \operatorname{sig}^{\gamma_{1}}(\zeta)- & \|\zeta\|_{2}>\zeta_{0} \\ \frac{\left\|S^{T} \Delta u\right\|_{1}+0.5 \Delta u^{T} \Delta u}{\|\zeta\|_{2}^{2}} \zeta+\Delta u & \|{ }^{2}{ }^{2}\end{cases}
$$

Where ' $\Delta u=\operatorname{sat}(u)-u$ ', ' $k_{1}$ ', ' $k_{2}$ ' and ' $\zeta_{0}$ ' are positive constants and ' $\operatorname{sig}^{\gamma_{1}}(\zeta)=\left[\operatorname{sig}^{\gamma_{1}}\left(\zeta_{1}\right), \operatorname{sig}^{\gamma_{1}}\left(\zeta_{2}\right), \operatorname{sig}^{\gamma_{1}}\left(\zeta_{3}\right)\right]^{T}$.

The Chen Control Law is given by (52).

$$
\begin{array}{r}
-u=-F-\alpha_{1} J_{B}^{*} \delta \dot{q}_{13}-\alpha_{2} J_{B}^{*} \dot{\beta}\left(\delta q_{13}\right)-k_{3} \zeta-\frac{1}{2} S \\
+u_{r}+u_{n}+u_{a}
\end{array}
$$

Where ' $u_{a}$ ' and ' $F$ ' are defined in (53) and (56) respectively.

$$
\begin{gathered}
u_{a}=-\hat{u} \frac{S}{\|S\|_{2}+\epsilon}, \epsilon=\frac{k_{0}}{1+\hat{u}} \\
\hat{u}=\hat{c}_{0}+\hat{c}_{1}\|\delta \omega\|_{2}+\hat{c}_{2}\|\delta \omega\|_{2}^{2} \\
\hat{c}_{n}=p_{n}\left(\|S\|_{2}\|\delta \omega\|_{2}^{n}-\chi_{n} \hat{c}_{n}\right), n=1,2,3 \\
F=-[\omega \times] J_{B}^{*} \omega+J_{B}^{*}\left([\delta \omega \times] C(\delta q) \omega_{d R}-C(\delta q) \dot{\omega}_{d R}\right)
\end{gathered}
$$

Equations for ' $u_{r}$ ' and ' $u_{n}$ ' are defined in (57) and (58) respectively.

$$
\begin{gathered}
u_{r}=-\tau_{1} S-\tau_{2} \operatorname{sig}^{\rho}(S) \\
u_{n}=-k_{4} s i g^{\gamma_{1}}(S)
\end{gathered}
$$

Where ' $\rho>0$ ', ' $\gamma_{1}<1$ ', ' $\tau_{1}$ ', ' $\tau_{2}$ ', ' $k_{3}$ ', ' $k_{4}$ ' are positive constants, and ' $k_{1}-\frac{1}{3} k_{3}^{2}-\frac{1}{2}>0$ '.

Chen defines the saturation function as (60).

$$
\begin{gathered}
\operatorname{sat}(u)=\left[\operatorname{sat}\left(u_{1}\right), \operatorname{sat}\left(u_{2}\right), \operatorname{sat}\left(u_{3}\right)\right]^{T} \\
\operatorname{sat}\left(u_{i}\right)= \begin{cases}\left(U_{\max }-a\right)+ & u_{i} \geq U_{\max }-a \\
\operatorname{atanh}\left(\frac{u_{i}-U_{\max }+a}{a}\right), & a-U_{\max }<u_{i} \\
u_{i}, & <U_{\max }-a \\
\left(a-U_{\max }\right)+ & \\
\operatorname{atanh}\left(\frac{u_{i}+U_{\max }-a}{a}\right), & u_{i} \leq a-U_{\max }\end{cases}
\end{gathered}
$$

Where ' $U_{\max }$ ' is the maximum control torque delivered by the reaction wheels, ' $a$ ' is positive constant, and ' $\mathrm{i}=1,2,3$ '.

\section{NUmERICAL Simulations}

Numerical simulations were carried out to perform regulation maneuvers for long angles. The block diagram shown in Fig. 3 describes how simulations were performed and the parameters taken from [15]. Parameters, initial conditions, and desired attitude can be seen in Tables I, II, and III, respectively.

The second-order Simpson's rule, shown in Appendix B, was used as the numerical integration method and the fourthorder Runge-Kutta algorithm was used to approximate the solution in differential equations. Simulation time was 200 seconds with a total of 100,000 iterations with a step of 0.2 milliseconds.

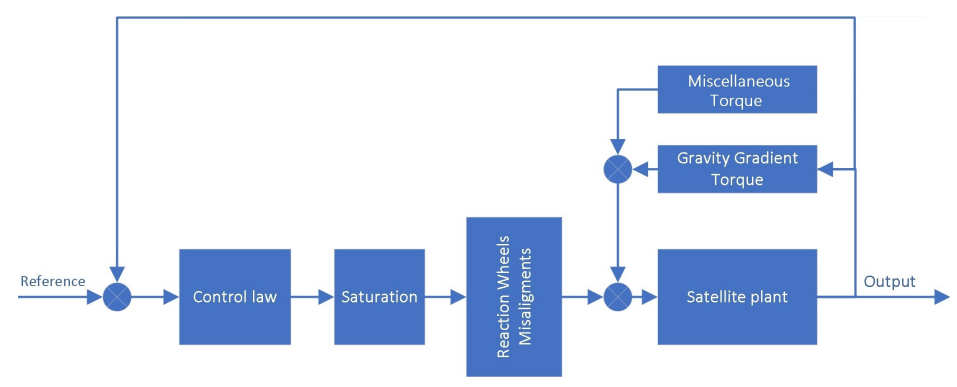

Fig. 3. Block diagram for a CubeSat subject to gravity gradient torque, and misalignment. The control law block may have adaptation algorithms depending on the simulated control law. 
TABLE I. PARAMETERS OF SATELLITE AND REACTION WHEELS

\begin{tabular}{|c|c|c|c|}
\hline \multicolumn{4}{|c|}{ Satellite Parameters } \\
\hline Parameter & & ues & \\
\hline CubeSat Inertia Tensor & $\tilde{J}_{B}=\left[\begin{array}{cc}8.46 & 1.1 \\
1.1 & 8.46 \\
1.5 & 1.6\end{array}\right.$ & $\left.\begin{array}{c}1.5 \\
1.6 \\
8.46\end{array}\right]$ & $\times 10^{-3}(\mathrm{Nm})$ \\
\hline Desired trajectory & \multicolumn{3}{|c|}{$\begin{array}{c}q_{d}=[0.2393,0.1893,0.0381,0.9515]^{T} \\
\omega_{d}=[0,0,0]^{T}(\mathrm{deg} / \mathrm{s})\end{array}$} \\
\hline Initial conditions & \multicolumn{3}{|c|}{$\begin{array}{c}q=[0,0,0,1]^{T} \\
\omega=[0,0,30]^{T}(\mathrm{deg} / \mathrm{s}) \\
\omega_{r w}=[0,0,30]^{T}(\mathrm{deg} / \mathrm{s})\end{array}$} \\
\hline \multicolumn{4}{|c|}{ Reaction wheels parameters } \\
\hline Parameter & \multicolumn{3}{|c|}{ Values } \\
\hline $\begin{array}{c}\text { Radius } \\
\text { Mass } \\
\text { Width } \\
\text { Maximum torque }\end{array}$ & \multicolumn{3}{|c|}{$\begin{array}{c}R_{r w}=4.3 \mathrm{~cm} \\
m_{r w}=25 \mathrm{~g} \\
d_{r w}=1.5 \mathrm{~cm} \\
1.343 \times 10^{-2}(\mathrm{Nm})\end{array}$} \\
\hline
\end{tabular}

TABLE II. PARAMETERS OF DISTURBANCES

\begin{tabular}{|c|c|}
\hline \multicolumn{2}{|c|}{ Gravity Gradient Torque } \\
\hline Parameter & Values \\
\hline$r_{c}$ & $6471 \times 10^{3} \mathrm{~m}$ \\
\hline \multicolumn{2}{|c|}{ Reaction wheel misalignments } \\
\hline Parameter & Values \\
\hline$\Delta \alpha_{1}, \Delta \alpha_{2}, \Delta \alpha_{3}$ & $3,-4,5 \mathrm{deg}$ \\
\hline$\Delta \beta_{1}, \Delta \beta_{2}, \Delta \beta_{3}$ & $10,-50,70 \mathrm{deg}$ \\
\hline
\end{tabular}

TABLE III. CONTROL PARAMETERS

\begin{tabular}{|c|c|}
\hline \multicolumn{2}{|c|}{ Quaternion Feedback Controller } \\
\hline Parameter & Values \\
\hline Calculated Inertia Tensor $^{\mathrm{a}}$ : & $J_{B}^{*}=I_{3}(\mathrm{Nm})$ \\
\hline $\mathrm{K}^{\mathrm{a}}, \mathrm{P}^{\mathrm{a}}:$ & $I_{3}, I_{3}$ \\
\hline Calculated Reaction wheels parameters & $\begin{array}{l}R_{r w}=4 \mathrm{~cm} \\
d_{r w}=1 \mathrm{~cm} \\
m_{r w}=20 \mathrm{~g}\end{array}$ \\
\hline \multicolumn{2}{|c|}{ Boskovic Robust Controller } \\
\hline Parameter & Values \\
\hline$\delta_{k}, \gamma$ & $0.01,0.001$ \\
\hline$U_{\max }$ & $1.343 \times 10^{-2} \mathrm{Nm}$ \\
\hline Initial condition & $K_{0}=1$ \\
\hline \multicolumn{2}{|c|}{ Dando Adaptive Controller } \\
\hline Parameter & Values \\
\hline Calculated Inertia Tensor $^{\mathrm{a}}$ & $J_{B}^{*}=I_{3}(\mathrm{Nm})$ \\
\hline$\lambda, \gamma, K d$ & $1,0.001,1$ \\
\hline Initial condition & $\theta_{0}=[1,1,1,0,0,0]^{T}$ \\
\hline \multicolumn{2}{|c|}{ Chen Robust Controller } \\
\hline Parameter & Values \\
\hline Calculated Inertia Tensor $^{a}$ & $J_{B}^{*}=I_{3}(\mathrm{Nm})$ \\
\hline$\alpha_{1}, \alpha_{2}, \eta$ & $1,0.5,0.0001$ \\
\hline$\gamma, \gamma_{1}, \rho$ & $0.6,0.7,0.7$ \\
\hline$k_{1}, k_{2}, k_{3}, k_{4}$ & $2,1,0.3,1$ \\
\hline$\tau_{1}, \tau_{2}, k_{0}$ & $10,10,0.0005$ \\
\hline$p_{0}, p_{1}, p_{2}$ & $0.1,0.1,0.1$ \\
\hline$\chi_{1}, \chi_{2}, \chi_{3}$ & $0.001,0.001,0.001$ \\
\hline$\zeta_{0}, a$ & $0.0001,0.5$ \\
\hline
\end{tabular}

\section{A. Attitude Regulation Maneuvers and Torque Magnitude Constraints}

The rest-to-rest attitude maneuver for a non-spinning CubeSat is simulated with all control laws subject to saturation with a maximum torque of $13.45 \times 10^{-3} \mathrm{Nm}$ emulating the physical limitations of the reaction wheels. Euler angles, angular rate error, and control torque are shown in Appendix C (Fig. 9, 10, 11 , and 12 ), respectively.

\section{B. Disturbances Torque Rejection}

In order to evaluate the Disturbance Torques Rejection, a miscellaneous disturbance torque was added as follows:

$$
T_{\text {mis }}=0.7[\sin (t), 2 \cos (2 t), 3 \sin (3 t)]^{T} \times 10^{-3} \mathrm{Nm}
$$

The results obtained were shown in Euler Angles since the attitude Quaternion does not have any physical sense. The conversion of Quaternions to Euler Angles was carried out using the asymmetric XYZ sequence. The results obtained are shown in Figures 4, 5, 6, and 7.
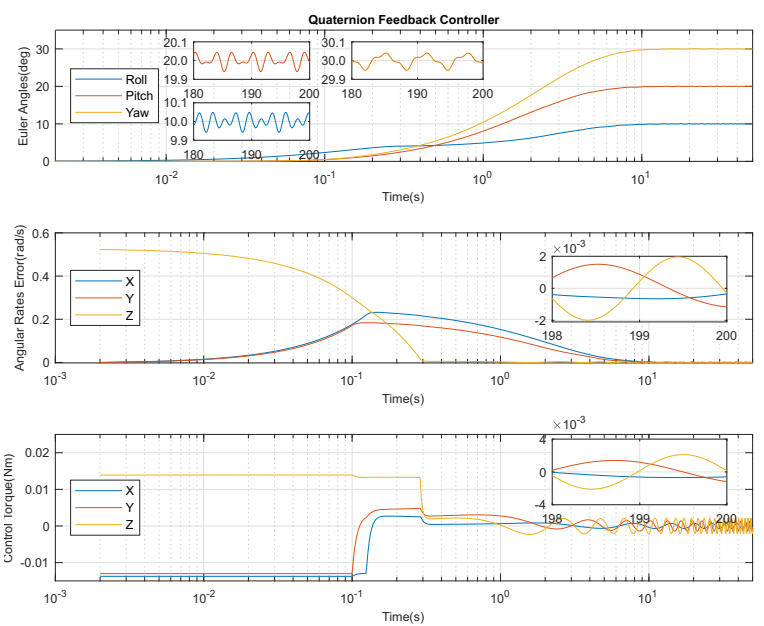

Fig. 4. Euler Angles and Angular Rate Errors produced by the miscellaneous disturbance torque in the Controller defined in (32) with a settling time (ts) of $6.81, \pm 0.04$ degrees in the Euler Angles oscillations, $\pm 1.9 \times 10^{-3}$ $\mathrm{rad} / \mathrm{s}$ oscillations in angular rates, and $\pm 2.1 \times 10^{-3} \mathrm{Nm}$ in Control Torque.

\section{Evaluation of Performance Between Laws of Control}

To compare the efficiency of the different control laws, several articles have been published [4][5][6]. Precision, computational cost, and stability are used as evaluation criteria in [20]. In [21], a performance index that considers both the thruster activity and the attitude tracking performance is used.

In this paper, five criteria of comparison have been used: EULERINT, ASCCT, settlement time at $5 \%\left(t_{s}\right)$, average computational cost $(\mathrm{O})$, and steady state error $\left(e_{s s}\right)$.

- $\quad$ Error Euler Angle Integration (EULERINT). Sidi [2] defines it as the integral of the error angle about the Euler axis of rotation. This is a quality indicator since it shows the accumulated angle error that the CubeSat travels to reach the desired position. The adapted formula to calculate it with Quartenions is described in (62). This parameter is similar to the attitude tracking performance metric defined in [21].

$$
\text { EULERINT }=\int_{0}^{T} 2 \cos ^{-1}\left(\delta q_{4}\right)
$$



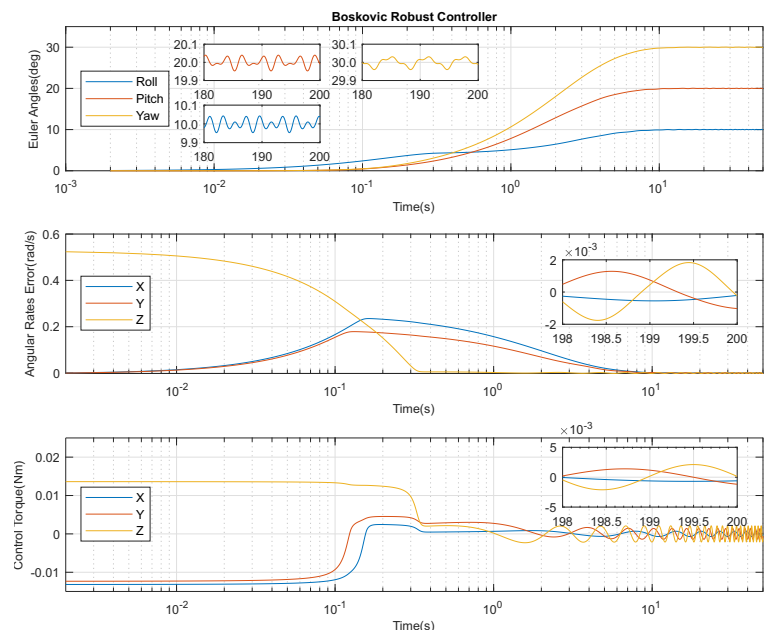

Fig. 5. Euler Angles and Angular Rate Errors produced by the miscellaneous disturbance torque in the Controller defined in (33) with a settling time (ts) of $6.44, \pm 0.04$ degrees in the Euler Angles oscillations, $\pm 1.8 \times 10^{-3}$ $\mathrm{rad} / \mathrm{s}$ oscillations in angular rates, and $\pm 2.1 \times 10^{-3} \mathrm{Nm}$ in Control Torque.
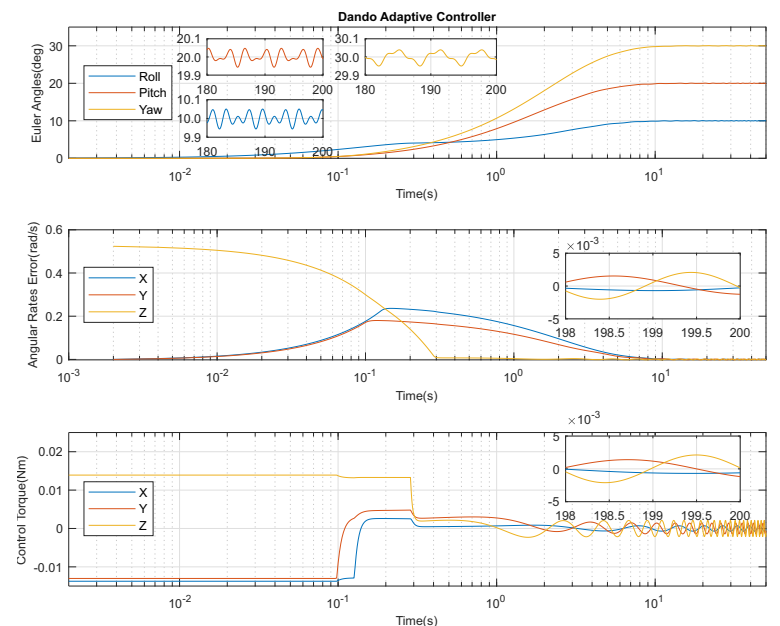

Fig. 6. Euler Angles and Angular Rate Errors produced by the miscellaneous disturbance torque in the Controller defined in (41) and (45) with a settling time (ts) of $6.48, \pm 0.05$ degrees in the Euler Angles oscillations, \pm 2.0 $\times 10^{-3} \mathrm{rad} / \mathrm{s}$ oscillations in angular rates, and $\pm 2.1 \times 10^{-3} \mathrm{Nm}$ in Control Torque.

- Average of Square of the Commanded Control Torque (ASCCT). It is defined by [12] as a measure of magnitude equivalent to the effective average torque exerted on the three satellite axes. This parameter is similar to the thruster activity performance metric defined in [21].

$$
A S C C T=\frac{1}{T} \int_{0}^{T}\|u(t)\|^{2} d t
$$

- Computational Cost $(\mathbf{O})$. The average time that the algorithm takes to calculate the new control command ' $u$ '.

The conducted simulations in Section VII-A were used to compare the control laws (27), (33), (41), and (52). The
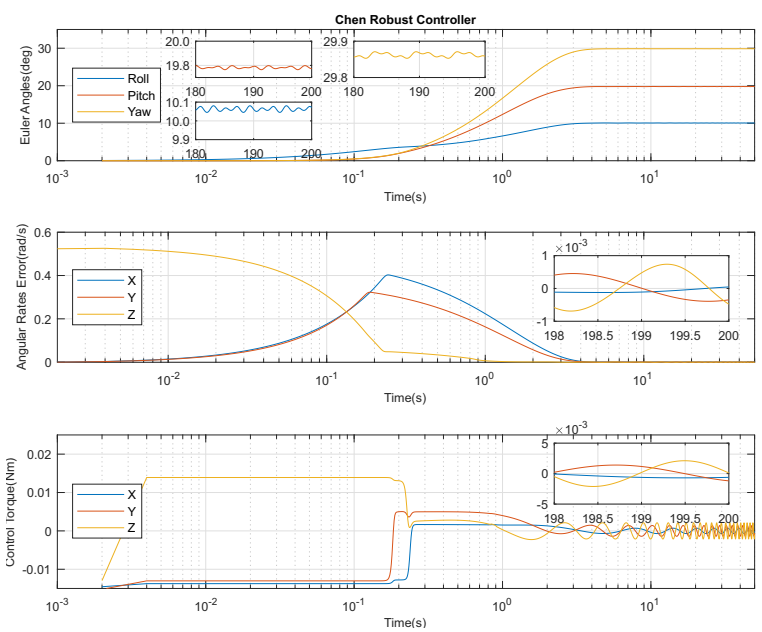

Fig. 7. Euler Angles and Angular Rate Errors produced by the miscellaneous disturbance torque in the Controller defined in (52) with a settling time (ts) of 2.75, \pm 0.01 degrees in the Euler Angles oscillations, 0.2 Euler Angles offset, $\pm 0.7 \times 10^{-3} \mathrm{rad} / \mathrm{s}$ oscillations in angular rates, and $\pm 2.1 \times 10^{-3}$ $\mathrm{Nm}$ in Control Torque.

calculated ASCCT and EULERINT are shown in Fig. 8.

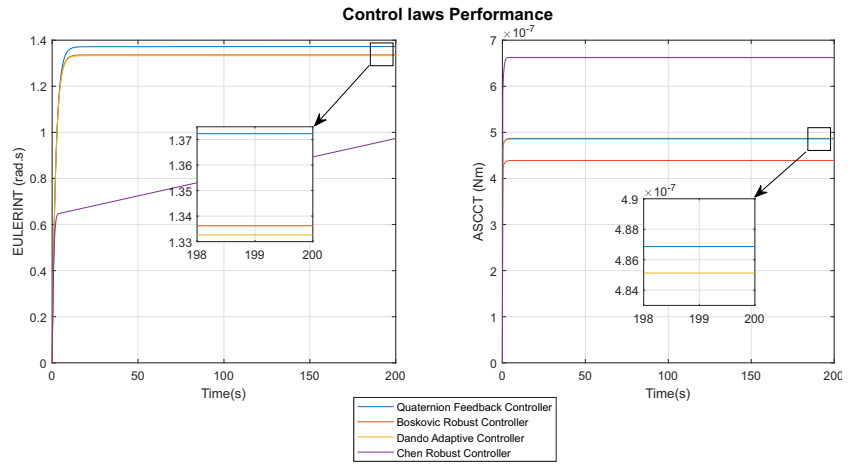

Fig. 8. EULERINT and ASCCT performance for control laws (27), (33),

(41), and (52) without effects of miscellaneous disturbance torque.

\section{RESULTS}

This section presents the results obtained in the numerical simulations developed in Section VII. The results obtained from Sections VII-A and VII-B are shown in Table IV and Table V, respectively.

TABLE IV. SiMULATION RESULTS WITHOUT EFFECTS OF MISCELLANEOUS TORQUES

\begin{tabular}{|c|c|c|c|c|c|}
\hline Controller & ts $\mathbf{( 5 \% )}$ & EULERINT & ASCCT & O & $e_{s s}$ \\
\hline Feedback & $6.83 \mathrm{~s}$ & 1.372 rad.s & $0.486 \mu$ N.m & $0.027 \mathrm{~ms}$ & $-0.1610^{-3}$ \\
\hline Boskovic & $6.32 \mathrm{~s}$ & 1.336 rad.s & $0.430 \mu$ N.m & $0.16 \mathrm{~ms}$ & $0.7110^{-6}$ \\
\hline Dando & $6.33 \mathrm{~s}$ & 1.333 rad.s & $0.485 \mu$ N.m & $0.20 \mathrm{~ms}$ & $0.9610^{-6}$ \\
\hline Chen & $2.75 \mathrm{~s}$ & Growing & $0.640 \mu \mathrm{N} . \mathrm{m}$ & $0.31 \mathrm{~ms}$ & -0.21 \\
\hline
\end{tabular}

The torque per gravity gradient for a balanced CubeSat of one unit is minimal. A magnitude of $[-3.14,6.44,-5.02]^{T} \times$ 
TABLE V. SiMULATION RESULTS WITH EFFECTS OF MISCELLANEOUS TORQUES

\begin{tabular}{|c|c|c|c|}
\hline Controller & ts (5\%) & Amplitude in Euler Angles oscillations & OFFSET \\
\hline Feedback & $6.81 \mathrm{~s}$ & $0.04 \mathrm{deg}$ & $0 \mathrm{deg}$ \\
\hline Boskovic & $6.44 \mathrm{~s}$ & $0.04 \mathrm{deg}$ & $0 \mathrm{deg}$ \\
\hline Dando & $6.48 \mathrm{~s}$ & $0.05 \mathrm{deg}$ & $0 \mathrm{deg}$ \\
\hline Chen & $2.75 \mathrm{~s}$ & $0.01 \mathrm{deg}$ & $0.2 \mathrm{deg}$ \\
\hline
\end{tabular}

$10^{-9} \mathrm{Nm}$ for the Gravity Gradient Torque is obtained as seen in Appendix D (Fig. 13).

\section{CONCLUSION}

In this article the simulation and comparison of four control laws in a CubeSat environment were carried out to find the most efficient control law that will be applied in the authors' future CubeSat projects.

The Quaternion Feedback Controller explained in Section VI-A is a basic algorithm that needs previous knowledge of the inertia tensor. Although, the miscellaneous torque rejection in this algorithm is not robust, it has an acceptable behavior rejecting constant inertia tensor uncertainties as can be seen in Fig. 4 and 9. However, the steady-state error is higher than the other robust algorithms and stability cannot be ensured in the case of control input constraints.

The Boskovic Robust Controller explained in Section VI-B is a robust algorithm that was developed considering the control input constraints so the authors ensure its global stability. To avoid the problem of chattering in the simulations, gain values for the adaptive parameter ' $k$ ' were chosen in order to not achieve convergence before the attitude and angular rate errors. As seen in Fig. 5, the controller does not reject the miscellaneous torque completely but the estimated computational cost is lower than the other robust algorithms as seen in Table IV. Moreover, the ASCCT parameter is the lowest but the EULERINT is only lower than the quaternion feedback algorithm.

The Dando Adaptive Controller law explained in Section VI-C is a robust algorithm that need a priori knowledge of the inertia tensor. Global stability cannot be ensured because this algorithm is not evaluated with control input constraints. Even though the saturation time to perform the maneuver is minimal, the behavior of the control law can be acceptable as seen in Fig. 6 and 11. The disturbance rejection is similar to Boskovic Controller but its steady state behavior is better than the other control laws.

The Chen Robust Controller law explained in Section VI-D is a robust controller that takes into account the control input constraints and needs a priori knowledge of the inertia matrix. Even though the asymptotic convergence in a finite time cannot be ensured as seen in Fig. 7 and 8, the disturbance rejection is better than the other controllers. In the simulations, the minimum steady-state is 0.02 degrees and the ASCCT torque needed to produce the rest-to-rest maneuver is the highest.

In order to choose the best control algorithm the priority was given to the steady-state error because it allows to perform precise attitude maneuvers. As a second evaluation criterion, the EULERINT parameter was chosen since it allows to perform a maneuver with the least possible error. Computational cost (O) was not consider a major factor as complex algorithms are not a challenge in modern micro-controllers. The settling time (ts) was not relevant for the chosen application.

Following these guidelines and according to the results obtained, it can be seen that the best performing control law for rest-to-rest maneuvers is the Boskovic Control law. This controller is capable of maintaining a steady-state error of $0.71 \times 10^{-6}$ degrees while rejecting disturbances caused by misalignment, gravity gradient, and miscellaneous torques.

\section{FUTURE WORK}

Further research will implement and compare the studied control laws in an air bearing testing platform as proposed in [15]. Also, it is interesting to evaluate the performance of these control laws under noise effects and uncertainties in the overall system. Moreover, based on [20], further work will be focused on the measurement of the current consumed by each reaction wheel and the overall electric potential in each maneuver.

\section{ACKNOWLEDGMENT}

The authors are grateful for the support provided by the Center for Acquisition and Processing of Satellite Data (CAPDS) located in the Pedro Paulet Astronomical and Aerospace Institute (IAAPP) of the Universidad Nacional de San Agustin of Arequipa.

\section{REFERENCES}

[1] J. Carnahan, "CubeSat Design Specification," CubeSat Program, Cal Poly SLO, 1999.

[2] M. J. Sidi, "Spacecraft Dynamics and Control," Cambridge University Press, 1997.

[3] J. E. Slotine et al., "Applied nonlinear control," Englewood Cliffs, NJ: Prentice hall, 1991.

[4] H. J. Marquez, "Nonlinear control systems. John Wiley \& Sons," 2003.

[5] Schaub, Hanspeter et al., "Feedback control law for variable speed control moment gyros," Journal of the Astronautical Sciences, 1998, vol. 46 , no 3, p. 307-328.

[6] A. J. Dando, "Robust Adaptive Control of Rigid Spacecraft Attitude Maneuvers," PhD Thesis, Queensland University of Technology, 2008.

[7] J. Ahmed, V. T. Coppola, and D. S. Bernstein, "Adaptive asymptotic tracking of spacecraft attitude motion with inertia matrix identification," Journal of Guidance, Control, and Dynamics, 1998, vol. 21, no 5, p. 684-691.

[8] S. Scarritt, "Nonlinear model reference adaptive control for satellite attitude tracking," in AIAA Guidance, Navigation and Control Conference and Exhibit. 2008. p. 7165.

[9] J. D. Boskovic, S. Li, and R. K. Mehra, "Robust tracking control design for spacecraft under control input saturation," Journal of Guidance, Control, and Dynamics, 2004, vol. 27, no 4, p. 627-633.

[10] H. Chen, S. Song, and Z. Zhu, "Robust finite-time attitude tracking control of rigid spacecraft under actuator saturation," International Journal of Control, Automation and Systems, 2018, vol. 16, no 1, p. $1-15$

[11] V. Carrara and H. K. Kuga, "Estimating friction parameters in reaction wheels for attitude control," Mathematical Problems in Engineering, 2013, vol. 2013

[12] B. Xiao et al., "Attitude tracking control of rigid spacecraft with actuator misalignment and fault," in IEEE Transactions on Control Systems Technology, 2013, vol. 21, no 6, p. 2360-2366.

[13] F. L. Markley and J. L. Crassidis, Fundamentals of spacecraft attitude determination and control. New York: Springer, 2014. 

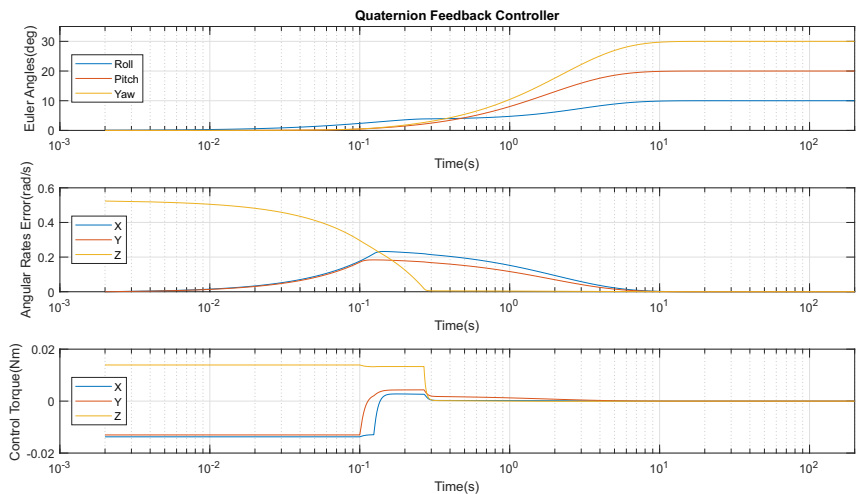

Fig. 9. Euler Angles, Angular Rate Errors and Control Torque produced by the Quaternion Feedback Controller defined in (27) with a settling time (ts) of $6.83 \mathrm{~s}$ and Average Computational Cost (O) of $0.027 \mathrm{~ms}$. The Control Torque saturation time was $267.5 \mathrm{~ms}$.
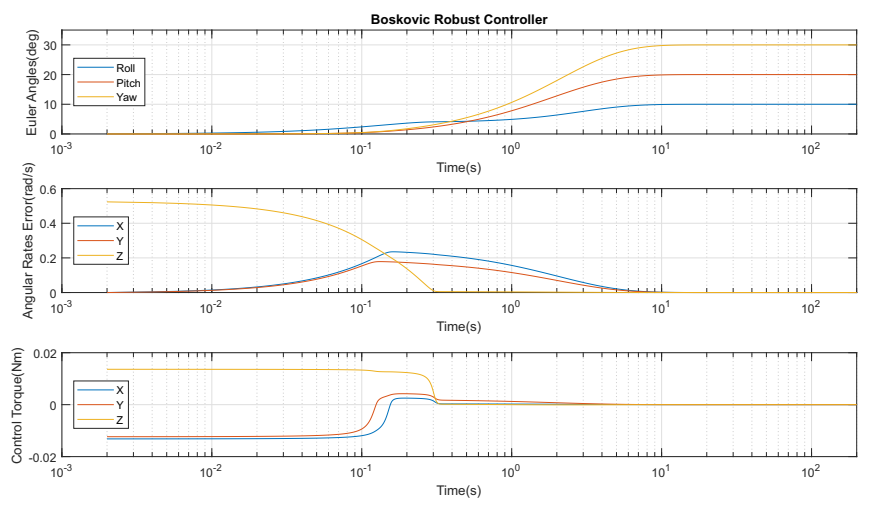

Fig. 10. Euler Angles, Angular Rate Errors and Control Torque produced by the Boskovic Robust Controller defined in (33) with a settling time (ts) of $6.32 \mathrm{~s}$ and average computational cost $(\mathrm{O})$ of $0.159 \mathrm{~ms}$. The Control Torque saturation time was $100.5 \mathrm{~ms}$.

[14] P. R. Yanyachi, "Modelagem e Control de Attitude de Satélites Artificiais com Apendices Flexiveis," Doctoral Thesis, Sao Paulo University, Brasil, 2005.

[15] H. Mamani, "Design and implementation of a testing platform for the picosatelite CubeSat attitude control and determination system," Undergraduate Thesis, National University of San Agustin, Perú, 2019.

[16] T. T. Arif, "A Decentralized Adaptive Control of Flexible Satellite," 2007 IEEE Aerospace Conference, Big Sky, MT, 2007, pp. 1-7.

[17] P. S. Pereira da Silva, F. S. Freitas and P.R. Y. Aco-Cardenas, "Flat systems, flat information and an application to attitude control," 1999 European Control Conference (ECC), Karlsruhe, 1999, pp. 3202-3207.

[18] R. Mukherjee and D. Chen, "Asymptotic stability theorem for autonomous systems," Journal of Guidance, Control, and Dynamics, 1993, vol. 16, no 5, p. 961-963.

[19] I. Kök, "Comparison and analysis of attitude control systems of a satellite using reaction wheel actuators," Dissertation, 2012.

[20] A. Bello et al., "Experimental comparison of attitude controllers for nanosatellites," in 8th European Conference for Aeronautics and Space Sciences, 2019

[21] N. Coulter and H. Moncayo, "Comparison of Optimal and Bioinspired Adaptive Control Laws for Spacecraft Sloshing Dynamics," Journal of Spacecraft and Rockets, 2020, vol. 57, no 1, p. 12-32.

\section{APPENDIX A}

Nomenclature used in this paper taken from Markley's book [13] .

\section{TABLE VI. NOMENCLATURE}

\begin{tabular}{|c|c|}
\hline Name & Symbol \\
\hline Attitude Quaternion & $q=\left[q_{1}, q_{2}, q_{3}, q_{4}\right]$ \\
\hline Quaternion vectorial part & $q_{13}$ \\
\hline Quaternion scalar part & $q_{4}$ \\
\hline Satellite angular velocity & $\omega$ \\
\hline Reaction wheels angular velocity & $\Omega$ \\
\hline Inertia tensor without reaction wheels & $\bar{J}_{B}$ \\
\hline Total inertia tensor & $J_{B}$ \\
\hline Perpendicular reaction wheel inertia & $J^{\perp}$ \\
\hline Parallel reaction wheel inertia & $J^{\|}$ \\
\hline Perpendicular reaction wheel inertia matrix & $J_{r w}$ \\
\hline External torques & $L$ \\
\hline Reaction wheels torques & $L_{r w}$ \\
\hline Gravity gradient torque & $T_{g g}$ \\
\hline Reaction wheel radio & $R_{r w}$ \\
\hline Reaction wheel thickness & $d_{r w}$ \\
\hline Reaction wheel mass & $m_{r w}$ \\
\hline Error Quaternion & $\delta q$ \\
\hline Angular velocity error & $\delta \omega$ \\
\hline Earth's gravitational coefficient & $\mu$ \\
\hline Distance from the center of Earth & $r_{c}$ \\
\hline
\end{tabular}

\section{APPENDIX B}

Numerical integration method used in Section VII: The second-order Simpson's rule.

$$
\int_{t_{1}}^{t_{2}} f(t) d t \approx \frac{d t}{6}\left[f\left(t_{1}\right)+4 f\left(\frac{t_{1}+t_{2}}{2}\right)+f\left(t_{2}\right)\right]
$$

\section{APPENDIX C}

The rest-to-rest attitude maneuver for a non-spinning CubeSat simulated in Section VII-A.
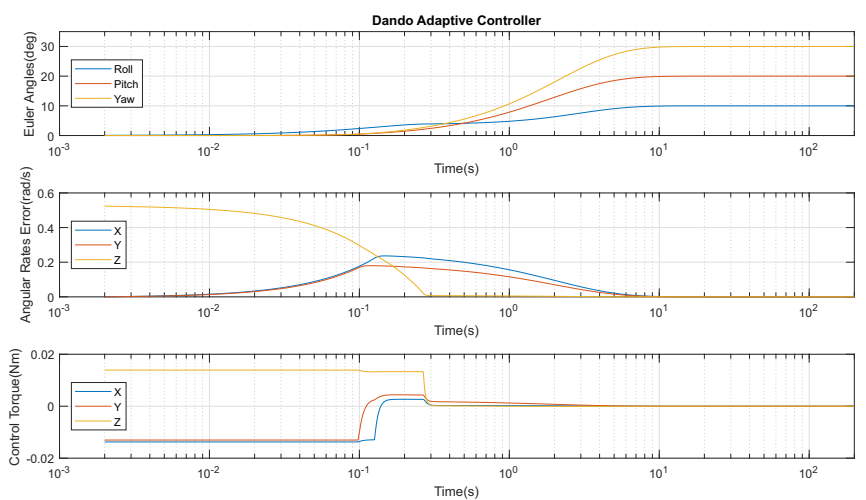

Fig. 11. Euler Angles, Angular Rate Errors and Control Torque produced by the Dando Adaptive Controller defined in (41) and (45) with a settling time (ts) of $6.33 \mathrm{~s}$ and average computational cost $(\mathrm{O})$ of $0.200 \mathrm{~ms}$. The Control Torque saturation time was $267 \mathrm{~ms}$. 

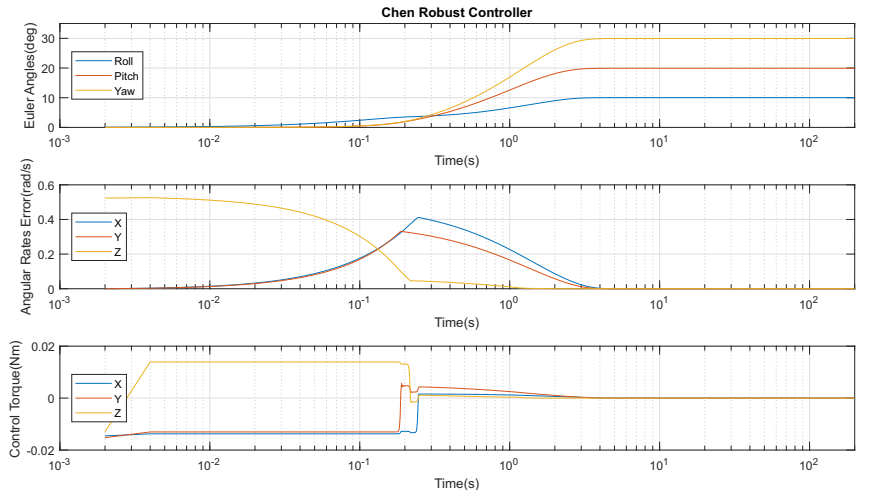

Fig. 12. Euler Angles, Angular Rate Errors, and Control Torque produced by the Chen Robust Controller defined in (52) with a settling time (ts) of $2.75 \mathrm{~s}$ and an average computational cost (O) of $0.309 \mathrm{~ms}$. The Control Torque saturation time was $235 \mathrm{~ms}$.
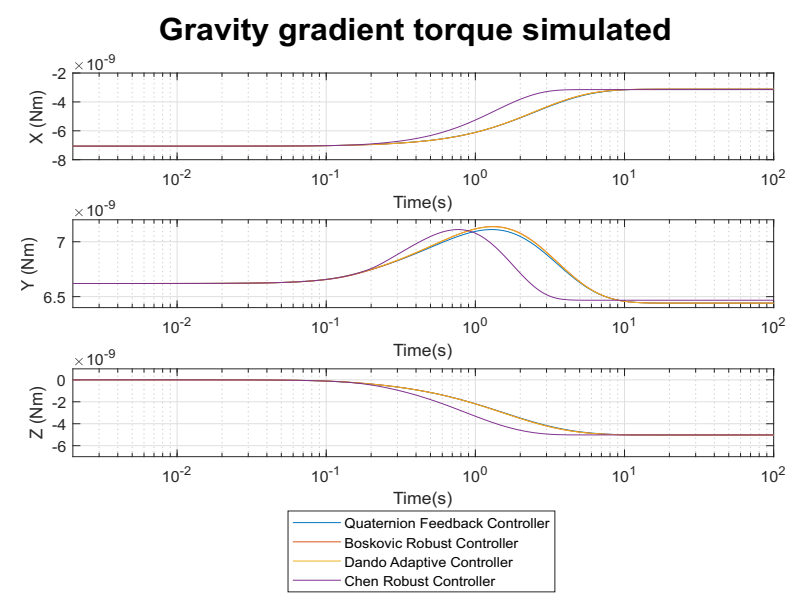

Fig. 13. Gravity gradient torque disturbance for a $100 \mathrm{Km}$ low orbit CubeSat.

\section{APPENDIX D}

Gravity Gradient Torque described by (17) in Section IV. 